Centre de Physique Théorique円 - CNRS - Luminy, Case 907

F-13288 Marseille Cedex 9 - France

\title{
FURTHER EXPERIMENTAL TESTS FOR SIMPLE RELATIONS BETWEEN UNPOLARIZED AND POLARIZED QUARK PARTON DISTRIBUTIONS \\ Claude BOURRELY and Jacques SOFFER
}

\begin{abstract}
Some simple relations between unpolarized and polarized quark parton distributions have direct experimental consequences which will be presented here. In particular, we will see that it is possible to relate the deep inelastic structure functions $F_{2}$ and $g_{1}$, both for proton and deuteron, in fair agreement with experimental data.
\end{abstract}

Key-Words : polarized parton densities, deep-inelastic scattering.

Number of figures : 3

August 1995

CPT-95/P.3224

anonymous ftp or gopher: cpt.univ-mrs.fr

${ }^{*}$ Unité Propre de Recherche 7061 
Deep inelastic scattering of polarized electrons and muons off polarized targets have been used to study the internal structure of the nucleon. The most abundant and accurate experimental information we have so far, concerns the so called $g_{1}$ spin structure function obtained with longitudinally polarized leptons on longitudinally polarized protons, deuterons and ${ }^{3} \mathrm{He}$ targets [1, 2, 3, 4, 5] which allow to get separately $g_{1}^{p}$ for protons and $g_{1}^{n}$ for neutrons. In the scaling limit, $g_{1}$ is a function of only the well known Bjorken variable $x=Q^{2} / 2 m_{p} \nu$, but when $Q^{2}$, the square of the four-momentum of the virtual photon of energy $\nu$, is finite, $g_{1}$ depends also on $Q^{2}$. So let us consider a situation with scaling violations and we recall that for protons one has

$$
g_{1}^{p}\left(x, Q^{2}\right)=\frac{1}{2}\left[\frac{4}{9} \Delta u\left(x, Q^{2}\right)+\frac{1}{9} \Delta d\left(x, Q^{2}\right)+\frac{1}{9} \Delta s\left(x, Q^{2}\right)\right],
$$

where for each flavor the polarized quark distribution

$$
\Delta q\left(x, Q^{2}\right)=q_{+}\left(x, Q^{2}\right)-q_{-}\left(x, Q^{2}\right),
$$

includes both quark and antiquark contributions. One obtains the neutron structure function $g_{1}^{n}\left(x, Q^{2}\right)$ by exchanging $u$ and $d$ and for the deuteron one has

$$
g_{1}^{d}=\frac{1}{2}\left(g_{1}^{p}+g_{1}^{n}\right)\left(1-3 / 2 \omega_{D}\right)
$$

where $\omega_{D}=0.05 \pm 0.01$ is the probability of the deuteron to be in a $D$-state. The measurements of polarized deep inelastic scattering yield in fact the spin asymmetry $A_{1}^{N}$ which is, to a good approximation,

$$
A_{1}^{N}\left(x, Q^{2}\right)=\frac{2 x g_{1}^{N}\left(x, Q^{2}\right)}{F_{2}^{N}\left(x, Q^{2}\right) /\left(1+R^{N}\left(x, Q^{2}\right)\right)},
$$

where $N=p, n$ or $d, F_{2}^{N}\left(x, Q^{2}\right)$ is the corresponding unpolarized structure function and $R^{N}=\left(F_{2}^{N}-2 x F_{1}^{N}\right) / 2 x F_{1}^{N}$ is small or zero in the leading order approximation. It is a well known experimental fact that $A_{1}^{N}\left(x, Q^{2}\right)$ is $Q^{2}$ independent, in the kinematical range so far explored. This means that numerator and denominator in Eq. (雨) have the same $Q^{2}$ dependence, within the present experimental accuracy. Clearly this observation, which has no sound theoretical justification, can be however used for phenomenological purposes, as we will see in what follows. 
Sometime ago [6, 7, 8] we have advocated the exclusion Pauli principle to postulate the existence of simple relations between unpolarized and polarized quark parton distributions. In particular, we have assumed [6, 7, 8] that

$$
\Delta u\left(x, Q^{2}\right)=u\left(x, Q^{2}\right)-d\left(x, Q^{2}\right)
$$

and also that the $s$ quarks are unpolarized (i.e. $\Delta s\left(x, Q^{2}\right)=0$ ). In addition, for the moment, if we ignore the contribution of $\Delta d\left(x, Q^{2}\right)$ in $g_{1}^{p}$, we obtain the simple relationship

$$
x g_{1}^{p}\left(x, Q^{2}\right)=\frac{2}{3}\left[F_{2}^{p}\left(x, Q^{2}\right)-F_{2}^{n}\left(x, Q^{2}\right)\right] .
$$

As a result for the proton case Eq. (14) reads

$$
A_{1}^{p}\left(x, Q^{2}\right)=\frac{4}{3}\left(1-r\left(x, Q^{2}\right)\right)\left(1+R^{p}\left(x, Q^{2}\right)\right)
$$

where $r\left(x, Q^{2}\right)=F_{2}^{n}\left(x, Q^{2}\right) / F_{2}^{p}\left(x, Q^{2}\right)$.

The NMC-NA37 experiment at CERN [9] has performed a very detailed measurement of $F_{2}^{p}$ and $F_{2}^{d}$ in a large kinematic range $0.006 \leq x \leq 0.6$ and $0.5 \leq Q^{2} \leq 55 \mathrm{GeV}^{2}$ and we have used their very new parametrization of the data [10] to calculate $r\left(x, Q^{2}\right)$. The best determination of $R^{p}\left(x, Q^{2}\right)$ in the kinematic range $0.1 \leq x \leq 0.9$ and $0.6 \leq Q^{2} \leq 20 \mathrm{GeV}^{2}$ can be found in [11]. Therefore one can make a numerical estimate of Eq. (7) which gives the contribution of $u$ quarks only to $A_{1}^{p}\left(x, Q^{2}\right)$. The results are shown in Fig. 1 for two $Q^{2}$ values, $Q^{2}=3$ and $10 \mathrm{GeV}^{2}$, together with the proton data from SMC[1] and SLAC[2]. It is remarkable to note that Eq. (7) leads to almost no $Q^{2}$ dependence, except for $x<0.1$ where it might be due to the lack of a precise knowledge of $R^{p}[12$. We also see that Eq. (7) gives the correct shape of the data, although it lies systematically above it. This is easily understood because we have neglected the contribution of the $d$ and $s$ quarks which are expected to be negative. Actually one could try to estimate it from the shift one observes with respect to the data, but one would not be able to disentangle the $d$ quarks from the $s$ quarks. So we propose instead, to use a different method which involves both proton and deuteron data. As we have seen above the $g_{1}$ structure functions for proton, neutron and deuteron are expressed in terms of the three polarized distributions $\Delta u, \Delta d$ and $\Delta s$. Clearly one can eliminate $\Delta s$ by considering $g_{1}^{p}-g_{1}^{n}$ and similarly, one can 
eliminate $\Delta d$ by considering $4 g_{1}^{p}-g_{1}^{n}$. If we now assume $\Delta s\left(x, Q^{2}\right)=0$ and using Eq. (4) we obtain as a consequence of Eq. (5)

$$
5 x g_{1}^{p}\left(x, Q^{2}\right)-\frac{4}{2-3 \omega_{D}} x g_{1}^{d}\left(x, Q^{2}\right)=5\left[F_{2}^{p}\left(x, Q^{2}\right)-F_{2}^{d}\left(x, Q^{2}\right)\right] .
$$

This simple relationship between $g_{1}^{p}, g_{1}^{d}$ and $F_{2}^{p}, F_{2}^{d}$ can be tested directly from experimental data. This test is shown in Fig. 2 where we have used for the l.h.s. of Eq. (8) the SMC data 11, 4] and the SLAC data 2, 3] on the $g_{1}$ 's and for the r.h.s. the NMC parametrization 10 for $F_{2}^{p}$ and $F_{2}^{d}$. The errors on the l.h.s. have been calculated by using the statistical errors only, added in quadrature. The estimated errors for the r.h.s. are represented by dashed lines on both sides of the full line which corresponds to the NMC parametrization. The test is indeed very well satisfied and gives, within the experimental uncertainty, a fairly good support to Eq. (5) and to the assumption $\Delta s\left(x, Q^{2}\right)=0$. Moreover if one takes the first moment of both sides of Eq. (8), one finds for the l.h.s. $0.588 \pm 0.054$ using the SLAC data or $0.605 \pm 0.074$ using the SMC data, whereas the r.h.s. gives $0.587 \pm 0.065$ using 113 .

Finally, let us consider the combination $4 g_{1}^{n}-g_{1}^{p}$ which eliminates $\Delta u$. Again if we assume $\Delta s\left(x, Q^{2}\right)=0$, by using Eq. (3) one finds

$$
\frac{16}{2-3 \omega_{D}} x g_{1}^{d}\left(x, Q^{2}\right)-5 x g_{1}^{p}\left(x, Q^{2}\right)=\frac{5}{6} x \Delta d\left(x, Q^{2}\right) .
$$

Now according to the arguments we have used in [8], one is led to assume that

$$
\Delta d\left(x, Q^{2}\right)=-\frac{1}{3} d_{v a l}\left(x, Q^{2}\right) .
$$

By combining Eqs. (9) and (10) one gets the following expression for $x d_{v a l}\left(x, Q^{2}\right)$

$$
x d_{v a l}\left(x, Q^{2}\right)=\frac{18}{5}\left[5 x g_{1}^{p}\left(x, q^{2}\right)-\frac{16}{2-3 \omega_{D}} x g_{1}^{d}\left(x, Q^{2}\right)\right]
$$

which also can be tested directly from the data on the $g_{1}$ 's. This test is shown in Fig.3 where, as previously, we have used for the r.h.s. of Eq. (11) the SMC data 1, 4] and the SLAC data 2, 3] on the $g_{1}$ 's and for the l.h.s. the following simple expression taken from 8 at $Q^{2}=3 \mathrm{GeV}^{2}$

$$
x d_{v a l}(x)=\frac{1.802 x^{0.738}}{e^{(x-0.231) / 0.092}+1} .
$$


The experimental uncertainty in this case is indeed rather large and, although this test does not lead to any inconsistency, we cannot claim that it gives a very strong support to Eq. (10).

To conclude we would like to stress that our simple relations between unpolarized and polarized quark parton distributions, Eqs. (5) and (10), are consistent with present experimental data. To establish them more firmly, one needs a substancial improvement of the data which is expected in the near future. Then it will be possible to decide if these relations are just a useful phenomenological guide or if they have a more fundamental significance.

\section{Acknowledgments}

We thank G. Altarelli and R. Windmolders for interesting comments. One of us (J.S.) is grateful for the kind hospitality at the Theory Division (CERN) where part of this work was done.

\section{References}

[1] Spin Muon Collaboration, D. Adams et al., Phys. Lett. B329, 339 (1994); Erratum, Phys. Lett. B339, 332 (1994).

[2] SLAC E143, K. Abe et al., Phys. Rev. Lett. 74, 346 (1995).

[3] SLAC E143, K. Abe et al., preprint SLAC-PUB95-6734, to appear in Phys. Rev. Lett..

[4] Spin Muon Collaboration, D. Adams et al., Phys. Lett. B357, 248 (1995).

[5] SLAC E142, D.L. Anthony et al., Phys. Lett. B259, 959 (1993).

[6] F. Buccella and J. Soffer, Mod. Phys. Lett., A8, 225 (1993); Europhysics Lett. 24, 165 (1993); Phys. Rev., D48, 5416 (1993).

[7] C. Bourrely and J. Soffer, Phys. Rev. D51, 2108 (1995).

[8] C. Bourrely and J. Soffer, Nucl. Phys. B445, 341 (1995). 
[9] New Muon Collaboration, P. Amandruz et al., Phys. Lett. B295, 159 (1992).

[10] We thank A. Bruell for providing us with the new NMC parametrization still preliminary. New Muon Collaboration, M. Arneodo et al., "Measurement of the proton and deuteron structure functions $F_{2}^{p}$ and $F_{2}^{d " \text {, }}$ to be submitted to Phys. Lett. B.

[11] L.W. Whitlow et al., Phys. Lett. B250, 193 (1990).

[12] A better parametrization of $R^{p}$ using CCFR neutrino data at small values of $x$ will be available soon, (A. Bodek, private communication).

[13] New Muon Collaboration, M. Arneodo et al., Phys. Rev. D50, R1 (1994).

\section{Figure Captions}

Fig.1 The spin asymmetry $A_{1}^{p}\left(x, Q^{2}\right)$ versus $x$ calculated from Eq. (7) at $Q^{2}=3 \mathrm{GeV}^{2}$ (dashed line) and $Q^{2}=10 \mathrm{GeV}^{2}$ (solid line) compared to the data at different $Q^{2}$ (open squares from [1] and full circles from [2]).

Fig.2 Experimental test of Eq. (8). Open squares are obtained from [1] and [4], full circles from [2] and [3]. Full line has been calculated from the parametrization of Ref. [10] at $Q^{2}=6 G_{e V}{ }^{2}$ and dashed lines are the corresponding estimated errors.

Fig.3 Experimental test of Eq. (11). Open squares are obtained from [1] and [1], full circles from [2] and [3]. Full line has been calculated using Eq. (12) from [8]. 


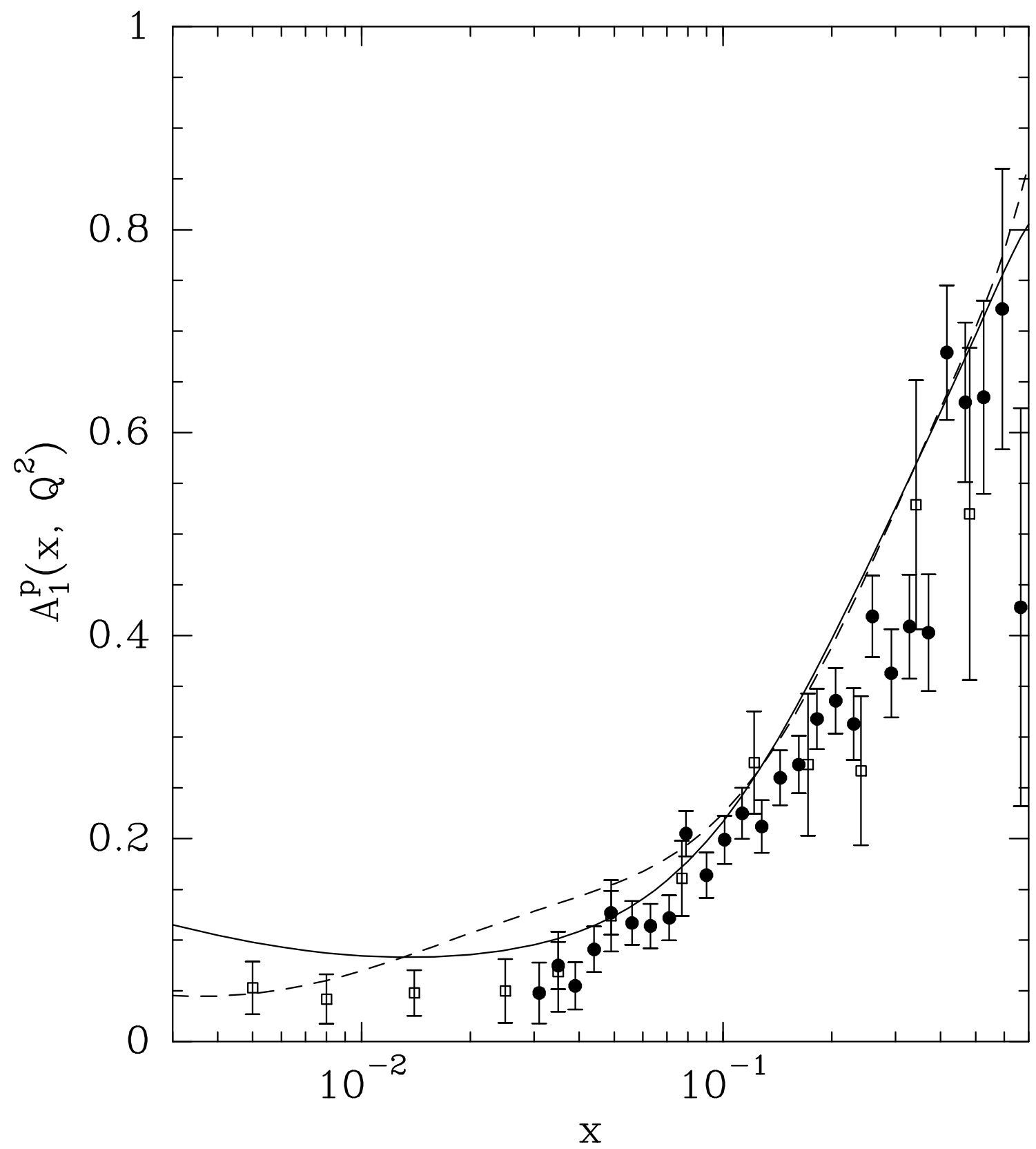

Fig 1 


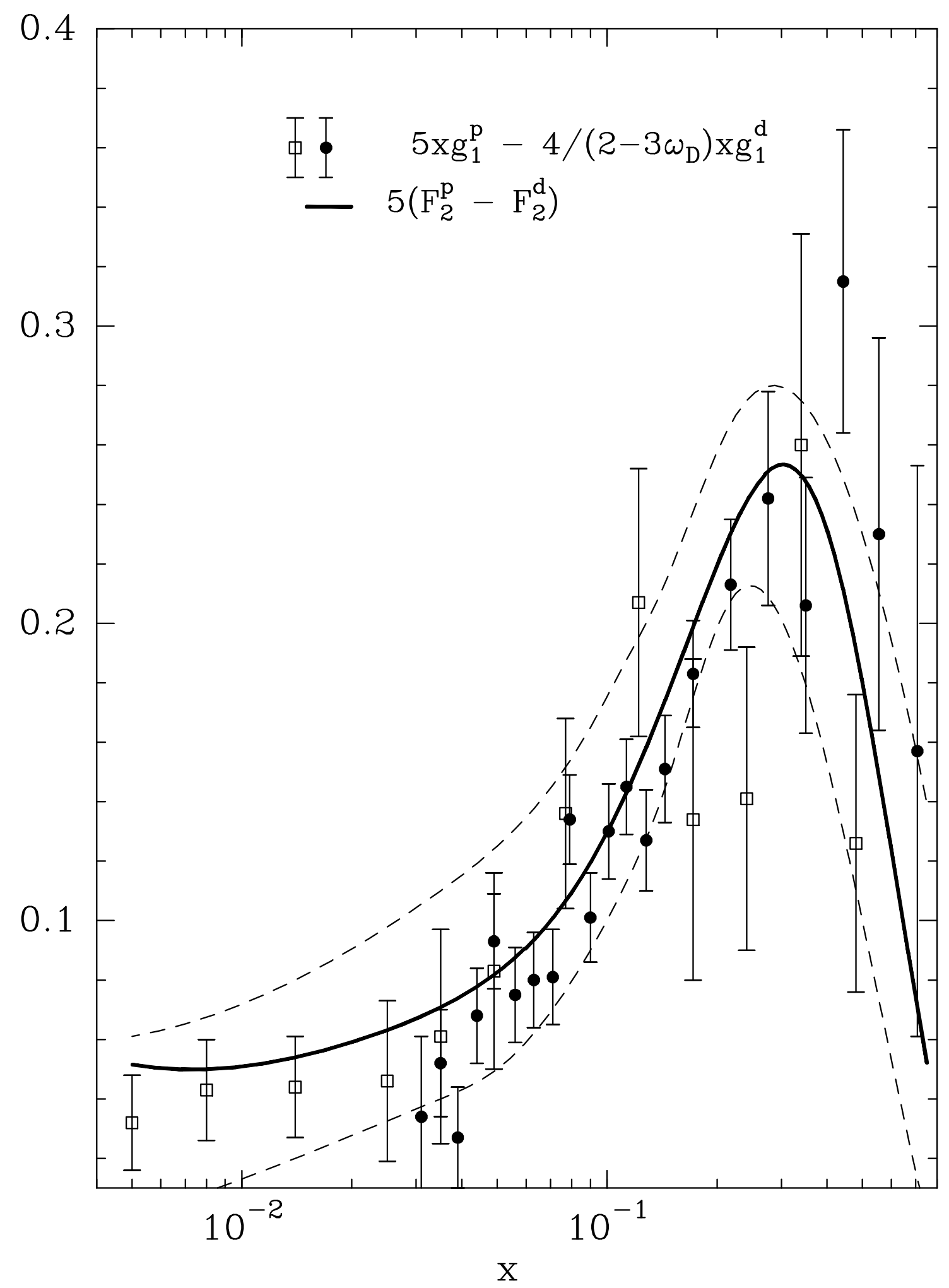

Fig 2 




Fig 3 